\title{
Small Crowders Slow Down Kinesin-1 Stepping by Hindering Motor Domain Diffusion
}

\author{
Krzysztof Sozański, ${ }^{1}$ Felix Ruhnow, ${ }^{2}$ Agnieszka Wiśniewska, ${ }^{1}$ Marcin Tabaka, ${ }^{1}$ Stefan Diez, ${ }^{2,}$ and Robert Hołyst ${ }^{1, \dagger}$ \\ ${ }^{1}$ Institute of Physical Chemistry, Polish Academy of Sciences, Kasprzaka 44/52, 01-224 Warsaw, Poland \\ ${ }^{2}$ B CUBE-Center for Molecular Bioengineering, Technische Universität Dresden, Arnoldstrasse 18, 01307 Dresden, Germany
}

(Received 7 February 2015; published 20 November 2015)

\begin{abstract}
The dimeric motor protein kinesin-1 moves processively along microtubules against forces of up to $7 \mathrm{pN}$. However, the mechanism of force generation is still debated. Here, we point to the crucial importance of diffusion of the tethered motor domain for the stepping of kinesin-1: small crowders stop the motor at a viscosity of $5 \mathrm{mPa} \cdot \mathrm{s}$ - corresponding to a hydrodynamic load in the sub-fN $\left(\sim 10^{-4} \mathrm{pN}\right)$ range — whereas large crowders have no impact even at viscosities above $100 \mathrm{mPa} \cdot \mathrm{s}$. This indicates that the scaledependent, effective viscosity experienced by the tethered motor domain is a key factor determining kinesin's functionality. Our results emphasize the role of diffusion in the kinesin-1 stepping mechanism and the general importance of the viscosity scaling paradigm in nanomechanics.
\end{abstract}

DOI: 10.1103/PhysRevLett.115.218102

PACS numbers: 87.16.Nn, 47.57.-s, 87.15.Vv

Within eukaryotic cells, active transport is driven by motor proteins such as kinesin-1 [1-3]. These molecular motors, stepping along microtubules (MTs), bind and translocate cargo ranging from nanometer-sized protein assemblies to micrometer-sized organelles. According to numerous in vitro studies, kinesin-1 motors perform continuous runs over distances of several micrometers at velocities of around $800 \mathrm{~nm} / \mathrm{s}$ [2,4-7]. Optical trapping experiments show that single kinesin-1 motors are capable of exerting forces of up to $7 \mathrm{pN}[4-6,8,9]$.

Force generation of kinesin-1 motors was also investigated by studying the motor-driven movement of gliding MTs [10] with attached micrometer-sized beads [11] in solutions of high viscosity. In these experiments crowding agents ("crowders") comprised polymers of high molecular weight. Kinesin slowed down with increasing viscosity due to the Stokes drag on the beads and MTs. No such effect was noted in a study of motility of unloaded kinesin in a polymer solution [12]. However, the impact of crowders similar in size to kinesin- 1 on its functionality has not been investigated. Such studies seem particularly appealing since molecular crowding plays a crucial role in many biologically important processes $[13,14]$ including diffusion $[15,16]$, macromolecular compaction $[17,18]$, enzymatic catalysis [19,20], or gene expression [21,22].

Here, we present experimental evidence favoring effective viscosity as the limiting factor for motility of individual kinesin-1 motors. Using crowding agents of low molecular weight $\left(M_{w}\right)$ we demonstrate that motion of motors can be stopped in solutions of effective viscosity of about $5 \mathrm{mPa} \cdot \mathrm{s}$. This is 5 orders of magnitude less than expected

Published by the American Physical Society under the terms of the Creative Commons Attribution 3.0 License. Further distribution of this work must maintain attribution to the author(s) and the published article's title, journal citation, and DOI. from Stokes' law, which predicts that a molecule of kinesin1 moving at $100 \mathrm{~nm} / \mathrm{s}$ would experience a stalling drag force in a medium of viscosity as high as $\sim 700 \mathrm{~Pa} \cdot \mathrm{s}$. In contrast, high- $M_{w}$ crowders have no effect on the motor velocity even above $100 \mathrm{mPa} \cdot \mathrm{s}$. We use the strongly supported model of kinesin stepping $[2,5,6,23,24]$, where the tethered (i.e., not bound to the MT) motor domain reaches the next binding site by a diffusional search mechanism. We explain the observed kinesin stall by hindered diffusion of the motor domain, employing the viscosity scaling paradigm [15,25-27] to explain the influence of the crowder size. We examine the role of depletion interactions [28,29] and include the notion of activation energy for diffusion [30,31].

Truncated, green fluorescent protein (GFP)-labeled kinesin-1 motors stepping along immobilized MTs were imaged using total internal reflection fluorescence (TIRF) microscopy (Fig. 1, room temperature $297 \pm 2 \mathrm{~K}$ ). The GFP was located at the $\mathrm{C}$ terminus of the protein, so that the motor domains and neck-linker regions were preserved. Viscosity of the solutions was tuned using molecular crowders of

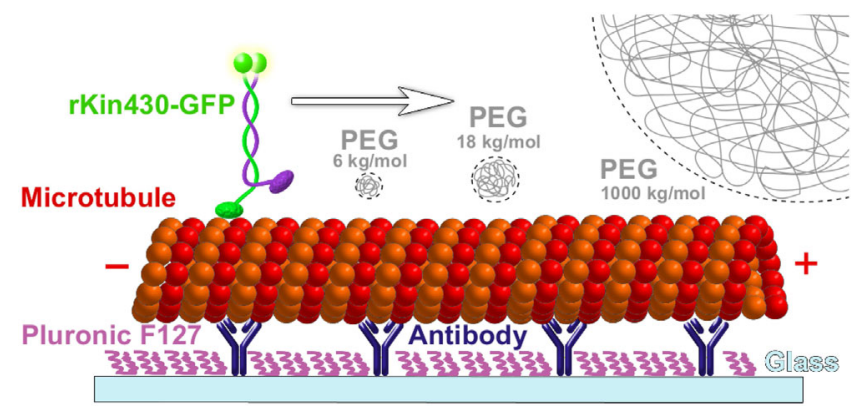

FIG. 1 (color online). Experimental setup of kinesin-1 stepping assays, drawn to scale. Hydrodynamic radii of the different PEGs are $2.17 \mathrm{~nm},(6 \mathrm{~kg} / \mathrm{mol}), 3.85 \mathrm{~nm}(18 \mathrm{~kg} / \mathrm{mol})$, and $35 \mathrm{~nm}$ (1000 kg/mol). 

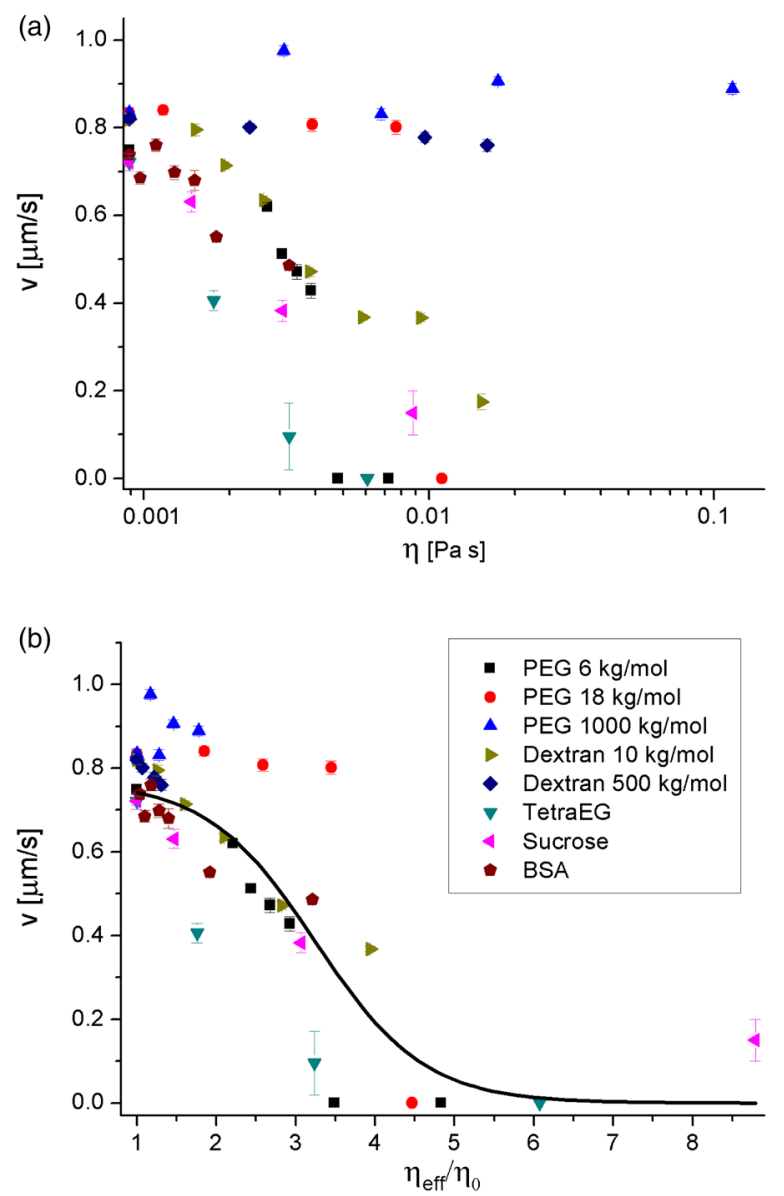

FIG. 2 (color online). Velocity of kinesin-1 motion along the MTs measured in stepping motility assays plotted against the following: (a) macroscopic solution viscosity and (b) effective viscosity experienced by a kinesin-1 motor domain [Eq. (1)], normalized by pure buffer viscosity. Error bars are standard deviation of the mean. Solid line is a fit of the load-dependent stepping model by Schnitzer et al. [42] (details in the text). Legend refers to both panels.

different sizes: polyethylene glycol (PEG; $M_{w}: 6,18$, and $1000 \mathrm{~kg} / \mathrm{mol}$ ), dextrans (approximately $M_{w} \quad 10$ and $500 \mathrm{~kg} / \mathrm{mol}$ ), tetraethylene glycol (TetraEG), sucrose, and bovine serum albumin (BSA). Stepping velocities of individual kinesin-1 motors were determined from TIRF image stacks using FIESTA [32]. Experimental details can be found in the Supplemental Material [33] and [40].

In the absence of crowders, at adenosine triphosphate (ATP) concentration of $1 \mathrm{mM}$, we observed average kinesin-1 velocities of about $800 \mathrm{~nm} / \mathrm{s}$, as expected $[4,7,41]$. No significant deviations from this value were observed in high- $M_{w}$ PEG $(1000 \mathrm{~kg} / \mathrm{mol})$ solutions, even at a concentration of $2 \%$, which corresponded to a macroscopic viscosity of $116 \mathrm{mPa} \cdot \mathrm{s}$ [Fig. 2(a)]. Analogous observations were made for $500 \mathrm{~kg} / \mathrm{mol}$ dextran solutions. Thus, even at high macroscopic viscosity originating from the presence of large crowders, the motility of kinesin- 1 was not impaired (in agreement with $[10,11]$ ). However, for solutions of low- $M_{w}$ polymers, TetraEG, or sucrose, we observed a decrease of kinesin-1 velocity upon increasing concentration of the crowders, up to an utter stall. A similar effect was observed for BSA solutions, although experiments at BSA concentrations sufficient for kinesin-1 stall could not be performed due to the protein's autofluorescence.

To properly assess the influence of crowders on diffusion of the tethered motor domain, we applied the viscosity scaling paradigm $[16,25-27,43,44]$. Viscosity experienced by nanoscopic probes in complex liquids is not equivalent to the macroscopic viscosity, but is a function of the probe size and characteristic length scales of the liquid. For instance, in solutions of polymers a loose mesh is formed, which dramatically increases the macroscopic viscosity. However, small probes can penetrate such mesh, experiencing a viscosity similar as in pure solvent. This phenomenon is quantified within scaling equations $[15,27,45]$ with effective viscosity $\eta_{\text {eff }}$ defined as

$$
\eta_{\mathrm{eff}}=\eta_{0} \exp \left[b\left(\frac{R_{\mathrm{eff}}}{\xi}\right)^{a}\right]
$$

where $\eta_{0}$ is the solvent viscosity, $\xi$ the correlation length $[15,46]$, and $a$ and $b$ are parameters of the order of unity. The effective radius, $R_{\text {eff }}^{-2}=R_{h}^{-2}+r_{p}^{-2}$, introduces the dependence of $\eta_{\text {eff }}$ on hydrodynamic radii of the probe and the crowders- $r_{p}$ and $R_{h}$, respectively.

We estimated $\eta_{\text {eff }}$ for the kinesin-1 motor domain by approximating it as a sphere of $r_{p}=2.5 \mathrm{~nm}$ and applying Eq. (1) (for scaling parameters see Table S2, Supplemental Material [33]). In the $1000 \mathrm{~kg} / \mathrm{mol}$ PEG or $500 \mathrm{~kg} / \mathrm{mol}$ dextran solutions, $\eta_{\text {eff }}$ for such a sphere is similar as in pure solvent (cf. viscosity scaling plots, Fig. S1, Supplemental Material [33]). Additionally, depletion effects lead to a decrease in polymer concentration near the MTs due to the lowering of the conformational entropy of the chains $[28,29,47-49]$. This effect extends over distances comparable to the hydrodynamic radius of the polymer coils (35 nm for $1000 \mathrm{~kg} / \mathrm{mol}$ PEG [50]). Therefore, the motor domain moves freely in this depletion zone, which facilitates its diffusion even more than expected from Eq. (1) (so that the already low $\eta_{\text {eff }}$ values calculated for these systems may still be overestimated).

The situation becomes qualitatively different for smaller crowders $\left(R_{h}\right.$ equal to $2.17,2.36,3.85$, and $4.0 \mathrm{~nm}$ for $6 \mathrm{~kg} / \mathrm{mol} \mathrm{PEG}, 10 \mathrm{~kg} / \mathrm{mol}$ dextran, $18 \mathrm{~kg} / \mathrm{mol} \mathrm{PEG}$, and BSA, respectively). According to Eq. (1), the difference between $\eta_{\text {eff }}$ and $\eta_{\text {macro }}$ diminishes with decreasing $R_{h}$. Also, the influence of the depletion effect diminishes. For TetraEG and sucrose, the hydrodynamic radii of which are $<1 \mathrm{~nm}$, both depletion and viscosity scaling effects become negligible.

As depicted in Fig. 2(b), a decrease of kinesin-1 velocity with increasing $\eta_{\text {eff }}$ is observed, with the motors stalling at about $5 \mathrm{mPa} \cdot \mathrm{s}$. With increasing $\eta_{\text {eff }}$ movement, events 

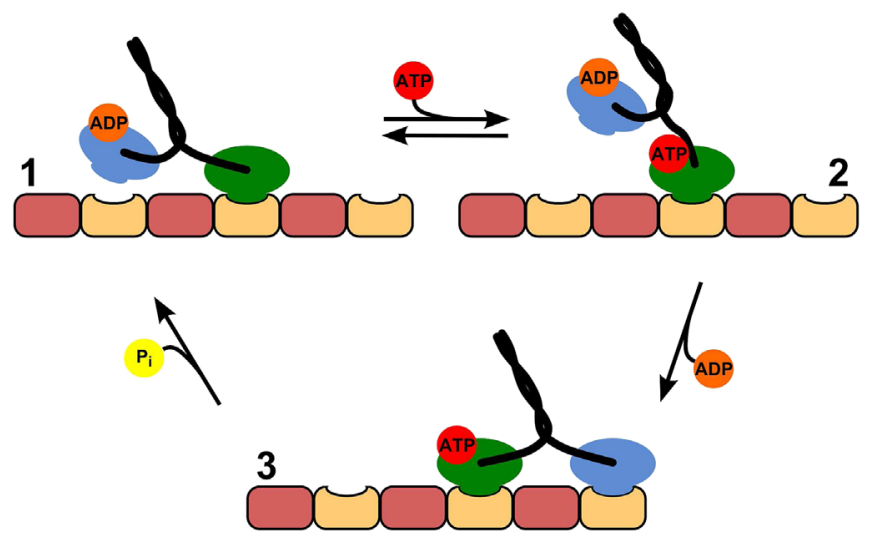

FIG. 3 (color online). Mechanism of kinesin-1 stepping [24]. The motor spends most of the time in the ATP-waiting state, $\mathbf{1}$. Binding of ATP to the MT-bound motor domain changes its conformation, enhancing the orientational freedom of the neck linker. The $\mathbf{1} \rightarrow \mathbf{2}$ reaction is reversible. As long as the MT-bound motor domain is in the ATP-bound state, the tethered domain can reach the subsequent binding site on the MT and release ADP. This movement, realized by diffusion, is constrained by viscosity and tension arising in the neck linker. When ATP is hydrolyzed, the MT-bound domain detaches from the MT, becoming the tethered one $(\mathbf{3} \rightarrow \mathbf{1})$.

become rare (despite no change in kinesin-1 concentration), up to the point where no motion is observed. Because of technical limitations (10 frames/ sec acquisition rate, $\sim 30 \mathrm{~nm}$ localization precision), we cannot directly discriminate single steps and assess their duration. However, over the observed time scales the motion-when it occurs-is generally characterized by a constant velocity (straight traces on kymographs), while any obvious stop-and-go events are excluded from the data analysis. In the further discussion we assume a steady processive movement of kinesin.

We use the strongly supported model of motion of kinesin-1 [24], schematically depicted in Fig. 3, to evaluate possible reasons for the loss of kinesin- 1 functionality with increasing $\eta_{\mathrm{eff}}$.

Hypothesis 1: Kinesin-1 aggregation.-Presence of a significant amount of polymer may lead to protein aggregation due to depletion forces and excluded volume effects $[14,51,52]$. We ruled out this possibility by fluorescence correlation spectroscopy experiments. We measured the time of diffusion of GFP-labeled kinesin-1 molecules through a focal volume in the presence of TetraEG (concentrations up to $50 \%$ ) and $18 \mathrm{~kg} / \mathrm{mol}$ PEG (up to $15 \%$ ). In both cases, the increase of diffusion time was directly proportional to the viscosity (see Supplemental Material, Fig. S2 [33]). This implies that the hydrodynamic radius of the protein remained constant irrespective of the crowder concentration. Furthermore, in TIRF images immobile proteins on the MTs were observed, which suggests that kinesin-1 binding to the MTs was not disabled.

Hypothesis 2: Osmotic depletion and disjoining pressure-Trapping of the motor domain on the
MT.-During each step, one of the motor domains detaches from the MT. This requires energy and is coupled to hydrolysis of an ATP molecule [24]. Work is performed against the disjoining pressure. It accounts not only for breaking the noncovalent bonds between kinesin and MT, but also for the depletion interactions $[28,29,51,52]$. These arise when free space is created between the motor domain and the MT surface, which is instantaneously filled by solvent molecules. However, large crowders cannot penetrate this space due to steric limitations and the arising gradient of osmotic pressure pushes the motor domain back to the MT surface.

We crudely estimated the depletion-dependent energetic barrier for the separation of the motor domain from the MT as comparable to $\Pi d V$. $\Pi$ is the difference in osmotic pressure [53] between the polymer solution and pure buffer; $d V$ corresponds to the volume of a cylinder of radius equal to the motor domain hydrodynamic radius $(2.5 \mathrm{~nm})$ and height of the polymer coil's diameter. The estimated energy barrier in a $10 \%$ solution of $18 \mathrm{~kg} / \mathrm{mol}$ PEG (where kinesin- 1 stalls) is $0.4 k_{B} T$, while in a $10 \%$ solution of $6 \mathrm{~kg} / \mathrm{mol}$ PEG (where kinesin-1 motility is retained) it is $5.4 k_{B} T$. The value for the smaller PEG suggests that the depletion-related energy barrier is not a hindering factor, because the kinesin- 1 motors still move under these conditions. Moreover, the calculated values (for all data see Table S3, Supplemental Material [33]) are well below the energy obtained from hydrolysis of a single ATP molecule (20-25 $\left.k_{B} T\right)$.

Hypothesis 3: Poor availability of ATP.—Combining Eq. (1) with the Stokes-Einstein equation, we estimated the diffusion rates of ATP molecules in the investigated solutions. The root mean square displacement of an ATP molecule during the dwell time of kinesin-1 $(10 \mathrm{~ms})$ is about $1.5 \mu \mathrm{m}$ for the $45 \%$ sucrose solution. At $1 \mathrm{mM}$ ATP (standard experimental conditions) there are about $6 \times 10^{5}$ ATP molecules per $\mu \mathrm{m}^{3}$; therefore, the influence of hindered ATP diffusion on kinesin-1 mobility seems highly improbable. Experiments performed at lowered ATP concentrations (range 50-1000 $\mu \mathrm{M}$ ) revealed that the ATP-related decrease of velocity is largely independent from the presence of the crowder (for plots see Fig. S3, Supplemental Material [33]). Fits of simple MichaelisMenten kinetics $\left[V=V_{\max }[\mathrm{ATP}] /\left(K_{M}+[\mathrm{ATP}]\right)\right.$, where $V$ is the kinesin turnover rate calculated by dividing the measured velocity by the step length of $8 \mathrm{~nm}$ ] gave kinetic parameters $V_{\max }=103.4 \pm 0.2 \mathrm{~s}^{-1}, K_{M}=311 \pm 2 \mu \mathrm{M}$ for control and $V_{\max }=52.4 \pm 1.4 \mathrm{~s}^{-1}, \quad K_{M}=474 \pm$ $54 \mu \mathrm{M}$ for $30 \%$ sucrose. The significant changes in the Michaelis constant $K_{M}$ and maximum rate $V_{\max }$ imply that crowding interferes with the mechanism of the reaction. Also, increasing [ATP] to $10 \mathrm{mM}$ (i.e., 10 times the standard concentration) did not cause an increase in stepping velocity either in control or in sucrose-crowded assays. 
Hypothesis 4: Hindered diffusion of the tethered motor domain.- Brownian motion of the tethered motor domain is crucial for kinesin-1 stepping [2,5,6,23]. A single step requires a displacement of the motor domain over a distance of $16 \mathrm{~nm}$, which should be covered in less that $4 \mu$ s (assuming a simplistic model of the motor domain equivalent to a sphere of hydrodynamic radius of $2.5 \mathrm{~nm}$ freely diffusing in a solution of $\eta_{\text {eff }}=5 \mathrm{mPa} \cdot \mathrm{s}$ ). This is far below the expected step duration (ms range) and should have no impact on the kinesin-1 stepping rate. However, the two motor domains are connected via the neck linker. To take a step, kinesin-1 needs to stretch the linker. Molecular dynamics simulations suggest that this causes a tension of $15-35 \mathrm{pN}[54,55]$. The linker is extended by $3.1 \pm 0.8 \mathrm{~nm}$. From an approximation of a wormlike chain, a spring constant $K \approx 5 \mathrm{mN} \mathrm{m}^{-1}$ is roughly estimated [54]. To assess the probability of stretching the neck linker so that the tethered motor domain can reach the binding site, we assumed a model describing a particle diffusing in a harmonic potential based on a simplified solution of the Smoluchowski equation [56]:

$$
P(x)=\left[\left(K /\left(2 \pi k_{B} T\right)\right]^{1 / 2} \exp \left[-K x^{2} /\left(2 k_{B} T\right)\right],\right.
$$

where $P$ is the probability of finding the particle at the distance $x$ away from the equilibrium position. Integrating this equation over $x>3.1 \mathrm{~nm}$ yields only a $\sim 0.03 \%$ probability of finding the leading motor domain at sufficient distance from the trailing one.

Diffusion is an activated process, characterized by an Arrhenius-like activation energy [30]. It has been shown that an increase of viscosity of a solution by the addition of a crowding agent is equivalent to an increase of the activation energy for diffusion [31]. It can be expressed as $\eta_{\text {eff }}=\eta_{0} \exp \left(\Delta E_{a} R^{-1} T^{-1}\right)$, where $\Delta E_{a}$ is the difference in activation energy for viscous flow between solution and pure solvent. A fivefold increase of the effective viscosity corresponds to a rise of $\Delta E_{a}$ by about $1.6 k_{B} T$. This energy is added to the $4 k_{B} T$ necessary to stretch the linker, according to theoretical estimations [54].

With higher effective viscosity, the energy barrier for motion rises. The energy of ATP hydrolysis is partially used to detach the trailing motor domain and induce conformational changes in the protein, while the rest of it is dissipated. The energy stored in the strained neck is also lost due to fast draining of momentum. Consequently, translation of the motor domain depends on the energy of thermal motions.

Transition between states $\mathbf{1}$ and $\mathbf{2}$ in Fig. 3 is reversible [24]. The rate of $\mathbf{2} \rightarrow \mathbf{3}$ transition is estimated to be $600 \mathrm{~s}^{-1}$ under optimal conditions [24]. The rate of ATP dissociation from the MT-bound domain $(\mathbf{2} \rightarrow \mathbf{1})$ is about $100 \mathrm{~s}^{-1}$. The increase of $\eta_{\text {eff }}$ upon addition of crowders hinders the $\mathbf{2} \rightarrow \mathbf{3}$ transition, while it does not affect the $\mathbf{2} \rightarrow \mathbf{1}$ transition. Therefore, ATP may dissociate before the step occurs. According to a study utilizing roadblocks [57], a stalled kinesin-1 motor dissociates from the MT after $\sim 0.4 \mathrm{~s}$. However, the depletion effect poses a barrier for kinesin diffusing away from the MT [12] and fast rebinding becomes probable. Because of the limited temporal and spatial resolution of imaging, such events are currently not possible to resolve, which also precludes accurate measurements of the run length.

Similar to the case of the viscosity-related hindrance of motion discussed herein, external load applied to the motor also interferes with the $\mathbf{2} \rightarrow \mathbf{3}$ transition. Schnitzer et al. [42] proposed an exponential dependence of the rate of this process, $k$, on the applied force $F: k=k_{0} \exp \left[-F \delta /\left(k_{B} T\right)\right]$, where $k_{0}$ refers to the zero load situation and $\delta$ is the length of the substep. This was incorporated into a MichaelisMenten model, including the catalytic turnover rate constant as well as the apparent second-order rate constant for ATP binding. The model was fitted to an extensive set of experimental data. Here, we apply this model as well as numeric parameters established in [42], leaving a single fitting parameter: the proportionality coefficient relating the external load on the motor to the intrinsic viscous load on the motor domain. The curve is used to describe the data in Fig. 2(b). We find that increasing $\eta_{\text {eff }} / \eta_{0}$ by one unit is equivalent in terms of kinesin slow-down to applying an external load of $\sim 1.5 \mathrm{pN}$. Such viscosity change causes an increase of the hydrodynamic drag on the protein only in the sub-fN range. This again implies that the viscosity increase does not merely enhance the viscous load against which kinesin works, but it interferes with the reaction mechanism.

We observed a general decrease in kinesin-1 velocity with increasing effective viscosity. Such effect was not noted in previous studies [10-12] because solutions of high- $M_{w}$ crowders were used; therefore, due to viscosity scaling and depletion, $\eta_{\text {eff }}$ experienced by the motor domain was similar as in a pure buffer. Hindrance of the diffusive motion of the tethered motor domain at high $\eta_{\text {eff }}$ disrupts the kinesin-1 mechanochemical cycle. So far, such a phenomenon was not observed in vivo- $\eta_{\text {eff }}$ experienced by the kinesin motor domain in an eukaryotic cell cytoplasm is about 2-4 $\mathrm{mPa} \cdot \mathrm{s}$ [15]. However, cytoplasm presents substantial inhomogeneities over space and time; it is intriguing to speculate that tuning the local viscosity could be a regulatory mechanism for cellular transport. This remains yet to be investigated. Our results strongly support the kinesin-1 stepping mechanism wherein transfer of the tethered motor domain to the next binding site is diffusive. They also emphasize the vital role of the viscosity scaling paradigm in biophysical studies.

The authors acknowledge financial support from the National Science Center (Maestro Grant No. 2011/02/A/ ST3/00143), the European Research Council (Starting Grant No. 242933, NanoTrans), the European Social Funds (Grant No. 100111059, MindNano and Grant No. 100107464, ChemIT), and the German Research Foundation (Cluster of Excellence Center for Advancing Electronics Dresden and the Heisenberg Program). 
${ }^{*}$ Corresponding author. diez@bcube-dresden.de

Corresponding author. rholyst@ichf.edu.pl

[1] G. Woehlke and M. Schliwa, Nat. Rev. Mol. Cell Biol. 1, 50 (2000).

[2] S. M. Block, Biophys. J. 92, 2986 (2007).

[3] N. Hirokawa and R. Takemura, Exp. Cell Res. 301, 50 (2004).

[4] K. Svoboda and S. M. Block, Cell 77, 773 (1994).

[5] M. Nishiyama, H. Higuchi, and T. Yanagida, Nat. Cell Biol. 4, 790 (2002).

[6] N. Carter and R. Cross, Nature (London) 435, 308 (2005).

[7] E. Toprak, A. Yildiz, M. T. Hoffman, S. S. Rosenfeld, and P. R. Selvin, Proc. Natl. Acad. Sci. U.S.A. 106, 12717 (2009).

[8] C. M. Coppin, D. W. Pierce, L. Hsu, and R. D. Vale, Proc. Natl. Acad. Sci. U.S.A. 94, 8539 (1997).

[9] H. Kojima, E. Muto, H. Higuchi, and T. Yanagida, Biophys. J. 73, 2012 (1997).

[10] A. J. Hunt, F. Gittes, and J. Howard, Biophys. J. 67, 766 (1994).

[11] J. Gagliano, M. Walb, B. Blaker, J. C. Macosko, and G. Holzwarth, Eur. Biophys. J. 39, 801 (2010).

[12] L. Conway and J. Ross, arXiv:1409.3455.

[13] R. J. Ellis, Trends Biochem. Sci. 26, 597 (2001).

[14] H.-X. Zhou, G. Rivas, and A. P. Minton, Annu. Rev. Biophys. 37, 375 (2008).

[15] T. Kalwarczyk, N. Ziębacz, A. Bielejewska, E. Zaboklicka, K. Koynov, J. Szymański, A. Wilk, A. Patkowski, J. Gapiński, H.-J. Butt, and R. Hołyst, Nano Lett. 11, 2157 (2011).

[16] M. B. Elowitz, M. G. Surette, P. E. Wolf, J. B. Stock, and S. Leibler, J. Bacteriol. 181, 197 (1999).

[17] J. Kim, C. Jeon, H. Jeong, Y. Jung, and B.-Y. Ha, Soft Matter 11, 1877 (2015).

[18] C. D. Chapman, S. Gorczyca, and R. M. RobertsonAnderson, Biophys. J. 108, 1220 (2015).

[19] A. P. Minton, J. Biol. Chem. 276, 10577 (2001).

[20] S. Hou, N. Ziebacz, T. Kalwarczyk, T. S. Kaminski, S. A. Wieczorek, and R. Holyst, Soft Matter 7, 3092 (2011).

[21] M. Tabaka, L. Sun, T. Kalwarczyk, and R. Hołyst, Soft Matter 9, 4386 (2013).

[22] C. Tan, S. Saurabh, M. P. Bruchez, R. Schwartz, and P. LeDuc, Nat. Nanotechnol. 8, 602 (2013).

[23] W. R. Schief and J. Howard, Curr. Opin. Cell Biol. 13, 19 (2001).

[24] B. E. Clancy, W. M. Behnke-Parks, J. O. L. Andreasson, S. S. Rosenfeld, and S. M. Block, Nat. Struct. Mol. Biol. 18, 1020 (2011).

[25] F. Brochard-Wyart and P. G. de Gennes, Eur. Phys. J. E 1, 93 (2000).

[26] A. Tuteja, M. E. Mackay, S. Narayanan, S. Asokan, and M. S. Wong, Nano Lett. 7, 1276 (2007).

[27] R. Hołyst, A. Bielejewska, J. Szymański, A. Wilk, A. Patkowski, J. Gapiński, A. Zywociński, T. Kalwarczyk, E. Kalwarczyk, M. Tabaka, N. Ziębacz, and S. A. Wieczorek, Phys. Chem. Chem. Phys. 11, 9025 (2009).

[28] Y. Mao, M. Cates, and H. Lekkerkerker, Physica A (Amsterdam) 222, 10 (1995).

[29] H. Lekkerkerker and R. Tuinier, Colloids and the Depletion Interaction, Lecture Notes in Physics (Springer, New York, 2011).

[30] R. E. Powell, W. E. Roseveare, and H. Eyring, Ind. Eng. Chem. 33, 430 (1941).

[31] K. Sozański, A. Wiśniewska, T. Kalwarczyk, and R. Hołyst, Phys. Rev. Lett. 111, 228301 (2013).
[32] F. Ruhnow, D. Zwicker, and S. Diez, Biophys. J. 100, 2820 (2011).

[33] See Supplemental Material at http://link.aps.org/ supplemental/10.1103/PhysRevLett.115.218102, which includes Refs. [34-39], for details on crowders used, viscosity scaling parameters and plots, experimental methods, FCS results, analysis of kinetic parameters, and estimeted values of depletion interactions.

[34] T. Kalwarczyk, K. Sozanski, S. Jakiela, A. Wisniewska, E. Kalwarczyk, K. Kryszczuk, S. Hou, and R. Holyst, Nanoscale 6, 10340 (2014).

[35] C. Gell, V. Bormuth, G. J. Brouhard, D. N. Cohen, S. Diez, C. T. Friel, J. Helenius, B. Nitzsche, H. Petzold, J. Ribbe, E. Schaffer, J. H. Stear, A. Trushko, V. Varga, P. O. Widlund, M. Zanic, and J. Howard, in Microtubules, in vitro, Methods in Cell Biology, Vol. 95, edited by L. Wilson and J. J. Correia (Academic Press, New York, 2010), pp. 221-245.

[36] S. Yadav, S. J. Shire, and D. S. Kalonia, Pharm. Res. 28, 1973 (2011).

[37] A. Wiśniewska, K. Sozański, T. Kalwarczyk, K. Kędra-Królik, C. Pieper, S. A. Wieczorek, S. Jakieła, J. Enderlein, and R. Hołyst, Polymer 55, 4651 (2014).

[38] Y. Rong, M.S. Thesis, Rutgers, The State University of New Jersey, 2008.

[39] K. R. Rogers, S. Weiss, I. Crevel, P. J. Brophy, M. Geeves, and R. Cross, EMBO J. 20, 5101 (2001).

[40] T. Korten, B. Nitzsche, C. Gell, F. Ruhnow, C. Leduc, and S. Diez, in Single Molecule Analysis, Methods in Molecular Biology, Vol. 783, edited by E. J. G. Peterman and G. J. L. Wuite (Humana Press, New York, 2011), pp. 121-137.

[41] T. Korten and S. Diez, Lab Chip 8, 1441 (2008).

[42] M. J. Schnitzer, K. Visscher, and S. M. Block, Nat. Cell Biol. 2, 718 (2000).

[43] H. K. Schachman and W. F. Harrington, J. Am. Chem. Soc. 74, 3965 (1952).

[44] G. D. J. Phillies, G. S. Ullmann, K. Ullmann, and T. H. Lin, J. Chem. Phys. 82, 5242 (1985).

[45] T. Kalwarczyk, M. Tabaka, and R. Hołyst, Bioinformatics 28, 2971 (2012).

[46] P.-G. de Gennes, Scaling Concepts In Polymer Physics (Cornell University Press, Ithaca, 1979).

[47] D. Ausserre, H. Hervet, and F. Rondelez, Macromolecules 19, 85 (1986).

[48] G. Fleer, A. Skvortsov, and R. Tuinier, Macromolecules 36, 7857 (2003).

[49] A. Ochab-Marcinek, S. A. Wieczorek, N. Ziebacz, and R. Holyst, Soft Matter 8, 11173 (2012).

[50] K. Devanand and J. C. Selser, Macromolecules 24, 5943 (1991).

[51] S. Asakura and F. Oosawa, J. Polym. Sci. 33, 183 (1958).

[52] A. Kulkarni, A. Chatterjee, K. Schweizer, and C. Zukoski, J. Chem. Phys. 113, 9863 (2000).

[53] N. P. Money, Plant Physiol. 91, 766 (1989).

[54] C. Hyeon and J. N. Onuchic, Proc. Natl. Acad. Sci. U.S.A. 104, 2175 (2007).

[55] V. Hariharan and W. O. Hancock, Cell. Mol. Bioeng. 2, 177 (2009).

[56] M. Lindner, G. Nir, A. Vivante, I. T. Young, and Y. Garini, Phys. Rev. E 87, 022716 (2013).

[57] R. Schneider, T. Korten, W. J. Walter, and S. Diez, Biophys. J. 108, 2249 (2015). 